\title{
New decidable upper bound of the second level in the Straubing-Thérien concatenation hierarchy of star-free languages
}

\author{
Jorge Almeida ${ }^{1}$ and Ondřej Klíma ${ }^{2}$ \\ ${ }^{1}$ Dep. Matemática / CMUP, Faculdade de Ciências, Universidade do Porto, Portugal \\ ${ }^{2}$ Dep. Mathematics and Statistics, Faculty of Science, Masaryk University, Brno, Czech Republic \\ received $15^{\text {th }}$ November 2009, revised 21 st July 2010, accepted $18^{\text {th }}$ August 2010.
}

\begin{abstract}
In a recent paper we gave a counterexample to a longstanding conjecture concerning the characterization of regular languages of level 2 in the Straubing-Thérien concatenation hierarchy of star-free languages. In that paper a new upper bound for the corresponding pseudovariety of monoids was implicitly given. In this paper we show that it is decidable whether a given monoid belongs to the new upper bound. We also prove that this new upper bound is incomparable with the previous upper bound.
\end{abstract}

Keywords: formal languages, regular languages, concatenation hierarchies, level two, star-free languages

\section{Introduction}

A well-known result due to Schützenberger [22] gives a syntactic characterization of star-free regular languages. This prompted both Eilenberg's identification of the combinatorial properties of classes of regular languages [8], leading to a natural correspondence between varieties of languages (excluding the empty word, i.e. +-languages, or possibly including it, i.e. $*$-languages) and pseudovarieties (respectively of semigroups or monoids), and Brzozowski's hierarchical construction of star-free +-languages. As acknowledged by Eilenberg [8, Chapter IX], the reason to stick to +-languages was to avoid technical difficulties with the empty word, but these were later overcome by Thérien [29] and Straubing [25], who also established a simple syntactic connection between the Brzozowski hierarchy and what came to be known as the Straubing-Thérien hierarchy. The hierarchies were later refined by Pin [14] by introducing intermediate (half) levels whose syntactic characterization depends on a stable quasiorder rather than just a congruence.

Starting from the trivial variety of languages, the levels of the refined Straubing-Thérien hierarchy are defined inductively by alternately taking polynomial and Boolean closures. While it is decidable whether a given regular language belongs to each of the levels $0,1 / 2,1$ and $3 / 2$, decidability remains an open problem for level 2 or higher. Via Eilenberg's correspondence, for the class $\mathcal{V}_{2}$ of all languages from the second level, the problem translates to decidability of membership of an arbitrary given finite monoid in 1365-8050 (c) 2010 Discrete Mathematics and Theoretical Computer Science (DMTCS), Nancy, France 
the corresponding pseudovariety of monoids $\mathrm{V}_{2}$. This is considered one of the main longstanding open problems in the algebraic theory of regular languages ( $c f$. [13]).

Pin and Straubing [15] showed that the languages from the second level $\mathcal{V}_{2}$ over a finite alphabet $A$ are the finite Boolean combinations of languages of the form $A_{0}^{*} a_{1} A_{1}^{*} a_{2} \cdots a_{k} A_{k}^{*}$, where the $a_{i}$ 's are letters and the $A_{j}$ 's are subsets of $A$. Work of several authors led to the conjecture that the equality $\mathrm{V}_{2}=\mathrm{B}_{1} \mathrm{~m}$ SI holds [18, 19, 26, 27, 28], where $\mathrm{m}$ is the Mal'cev product [13, Section 6], $\mathrm{B}_{1}$ is the pseudovariety of finite semigroups corresponding to the +-variety of languages of dot-depth one, and $\mathrm{SI}$ is the pseudovariety of finite semilattices. Indeed, Straubing [26, 27] established that the classes contain the same 2-generated monoids and Cowan [6, 可 that they contain the same inverse monoids, while Pin and Weil [18, Theorem 5.9] proved that a similar equality holds for all half levels.

In the recent paper [3] we disproved the equality $V_{2}=B_{1}$ m SI. We discovered certain new pseudoidentities which are satisfied by members of $\mathrm{V}_{2}$ and we showed an example of a monoid $M \in \mathrm{B}_{1}$ m $\mathrm{SI}$ which does not satisfy one of these pseudoidentities. The new pseudoidentities are all those of the form $u^{\omega}=u^{\omega} v u^{\omega}$ where $u$ and $v$ are pseudowords such that $\mathrm{V}_{3 / 2}$ satisfies the inequality $u \leq v$; here $\mathrm{V}_{3 / 2}$ is the pseudovariety of ordered monoids corresponding to level $3 / 2$ in the Straubing-Thérien hierarchy. These new pseudoidentities define a pseudovariety of finite monoids $F$. Now the results from [3] can be summarized in the following way: $\mathrm{V}_{2} \subseteq \mathrm{F}, M \in \mathrm{B}_{1} m \mathrm{SI}$, and $M \notin \mathrm{F}$, so that $M \notin \mathrm{V}_{2}$. This implies $\mathrm{B}_{1}$ (m) SI $\nsubseteq \mathrm{F}$ and $\mathrm{V}_{2} \neq \mathrm{B}_{1}$ m $\mathrm{SI}$.

In this paper we also provide an example of a monoid which belongs to $\mathrm{F}$ and does not belong to $B_{1}(m)$ SI, and so we also have $F \nsubseteq B_{1}(m)$ SI. Hence the two upper bounds $B_{1}(m) S I$ and $F$ are incomparable and we get a new tighter upper bound for the class $V_{2}$, namely $\left(B_{1}(m) S I\right) \cap F$. One can hope that the inclusion $\mathrm{V}_{2} \subseteq\left(\mathrm{B}_{1} \mathrm{~m} \mathrm{SI}\right) \cap \mathrm{F}$ turns out to be an equality. This equality would solve the main problem if one can show that the membership problem for the pseudovariety $\left(B_{1}(m) S I\right) \cap F$ is decidable. The main contribution of this paper is the decidability of the membership problem for the pseudovariety $F$ from which the decidability of the membership problem for $\left(B_{1} ; m S I\right) \cap F$ follows.

Perhaps surprisingly, the membership problem for the pseudovariety $F$ is not easy to solve. When we want to test whether the new pseudoidentities are satisfied in a given finite monoid $M$ the following relation plays a crucial role. For a finite monoid $M$ we define a relation $\tau_{3 / 2}(M) \subseteq M \times M$ by the rule $(s, t) \in \tau_{3 / 2}(M)$ if there is a pair of pseudowords $u, v$ such that $\mathrm{\bigvee}_{3 / 2} \models u \leq v$ and an evaluation $\varphi$ such that $\varphi(u)=s, \varphi(v)=t$. Now we see that $M \in \mathrm{F}$ if and only if for every $(s, t) \in \tau_{3 / 2}(M)$ we have $s^{\omega}=s^{\omega} t s^{\omega}$. The latter condition is easy to test whenever we know the relation $\tau_{3 / 2}(M)$. Unfortunately, we do not know whether it is possible to compute this relation in general. Our solution of the membership problem is based on the trick that it is enough to compute the transitive closure of $\tau_{3 / 2}(M)$, because the condition $s^{\omega}=s^{\omega} t s^{\omega}$ is satisfied for all pairs $(s, t)$ from $\tau_{3 / 2}(M)$ if and only if the condition is satisfied for all pairs $(s, t)$ from the transitive closure of $\tau_{3 / 2}(M)$.

The paper is organized as follows. In Section 2, we recall a few preliminaries and notation. Then in Section 3 we introduce the relation $\tau_{3 / 2}(M)$ and some other equivalent descriptions of this relation. Section $\bigoplus$ is devoted to the formal definition of the new upper bound $\mathrm{F}$ and the trick concerning the transitive closure of the relation $\tau_{3 / 2}(M)$. In Section 5 we prove the difficult part of the crucial characterization of the transitive closure of the relation $\tau_{3 / 2}(M)$. Here we apply the Factorization Forest Theorem [23]. The decidability of $F$ is also established, which achieves the purpose of this paper. Section 6 presents an example of a monoid which shows that $\mathrm{F} \nsubseteq \mathrm{B}_{1}$ im SI. 


\section{Preliminaries}

\subsection{Stable quasiorders}

In this paper the key notion is stable quasiorder in monoids. Here we recall some easy observations and we fix notation. Note that in this paper a monoid is either finite or a free (profinite) monoid.

For an arbitrary set $M$ we say that a relation $R$, i.e. a subset of $M \times M$, is a quasiorder if and only if it is reflexive and transitive. Such a relation $R$ determines an equivalence relation

$$
R^{e}=R \cap R^{-1}=\{(s, t) \in M \times M \mid(s, t) \in R \text { and }(t, s) \in R\} .
$$

Then we can consider the factor set $M / R^{e}$ which is naturally ordered by the relation $R$, i.e. $s R^{e} \leq t R^{e}$ if and only if $(s, t) \in R$. We will denote this ordered set $\left(M / R^{e}, \leq\right)$ simply by $M / R$. The number of classes in $M / R$ is called the index of $R$.

If we take an arbitrary relation $R$ on $M$ then we can consider the smallest transitive relation containing $R$, so-called transitive closure, namely $R^{*}=\bigcup_{i \in \mathbb{N}} R^{i}$, where $R^{i}$ is the composition of $i$ copies of $R$. More formally, for each $i \in \mathbb{N}$ we define

$$
R^{i}=\left\{(s, t) \in M \times M \mid \text { there exist } s_{0}, s_{1}, \ldots, s_{i} \in M \text { such that } s=s_{0} R s_{1} R s_{2} R \cdots R s_{i}=t\right\} .
$$

Note that if the original relation $R$ is reflexive then we have $R^{i} \subseteq R^{j}$ whenever $i \leq j$. This implies that for two arbitrary pairs $\left(s_{1}, s_{2}\right),\left(t_{1}, t_{2}\right) \in R^{*}$ there is an exponent $i$ such that $\left(s_{1}, s_{2}\right),\left(t_{1}, t_{2}\right) \in R^{i}$.

For an arbitrary monoid $M$ we say that a relation $R$ is stable if for all $s_{1}, s_{2}, t_{1}, t_{2} \in M$ we have

$$
\left(\left(s_{1}, s_{2}\right) \in R \text { and }\left(t_{1}, t_{2}\right) \in R\right) \text { implies }\left(s_{1} t_{1}, s_{2} t_{2}\right) \in R .
$$

In other words, the relation $R$ is stable if and only if $R$ is a subsemigroup of $M \times M$. In some papers a stable relation $R$ on a monoid $M$ is defined by the following condition

$$
(s, t) \in R \text { implies }((s z, t z) \in R \text { and }(z s, z t) \in R)
$$

for all $s, t, z \in M$. Note that (2.2) follows from (2.1) when $R$ is a reflexive relation. On the other hand if $R$ is a transitive relation then (2.1) is a consequence of (2.2).

If $R$ is a stable reflexive relation on a monoid $M$ then the transitive closure $R^{*}$ is a stable quasiorder on $M$. Indeed, for each $i$ the relation $R^{i}$ is a stable relation on $M$ and the reflexivity of $R$ implies the claim. This basic observation means that if we have a relation $R$ on $M$ and we want to construct the smallest stable quasiorder containing $R$ we can first extend $R$ to a reflexive relation, which we then use to generate a submonoid in $M \times M$, and finally we take the transitive closure.

\subsection{Free profinite monoid}

According to Reiterman's Theorem [20], pseudovarieties of algebras are defined by pseudoidentities, that is formal equalities of implicit operations. In the case of interest for this paper, implicit operations are operations whose interpretation in finite monoids commutes with homomorphisms. Reiterman's Theorem has been extended independently by Molchanov [11] (see also [12]), via the nonstandard approach, and by Pin and Weil [17] to first order structures, the latter having particularly in mind pseudovarieties of ordered monoids, for which the equality (of implicit operations) is replaced by the order relation. By endowing 
all unordered monoids from a given pseudovariety $\mathrm{V}$ by all possible stable partial orders, one obtains a pseudovariety of ordered monoids, which is identified with $\mathrm{V}$.

Simple examples of implicit operations are the so-called explicit operations, given by words, and the $\omega$-power, which associates to each element $s$ of a finite monoid its unique idempotent power $s^{\omega}=s^{n}$ $(n>0)$.

The implicit operations over a fixed finite set $A$ can be viewed as the elements of the projective limit $F_{A}$ of all $A$-generated finite monoids, which is the structure one is led to consider when trying to identify the "most general" such monoid. Here, finite monoids are viewed as topological monoids under the discrete topology. In general, projective limits of finite monoids are called profinite monoids. They may also be characterized as compact totally disconnected monoids. It turns out that $F_{A}$ is the free profinite monoid on the set $A$, in which the discrete submonoid generated by $A$, whose elements are the explicit operations, is a free monoid and thus is identified with $A^{*}$. Thus, elements of $F_{A}$ become a generalization of usual words, which prompts calling them alternatively pseudowords. See [1, 2] for details.

Denote by $P_{A}$ the monoid of all subsets of $A$ under the union operation. The mapping that sends each letter $a \in A$ to the singleton $\{a\}$ extends to a unique continuous homomorphism $\alpha: F_{A} \rightarrow P_{A}$. Its restriction to $A^{*}$ is the usual content function and, more generally, a letter $a \in A$ belongs to $\alpha(u)$ for a given pseudoword $u \in F_{A}$ if and only if there is a factorization of the form $u=x a y$ with $x, y \in F_{A}$ (cf. [1]. Section 8.1]). For this reason, $\alpha$ is also called the content function.

Note that $P_{A}$ is a semilattice, i.e. a commutative and idempotent monoid. Moreover, for $u, v \in F_{A}$, we have $\mathrm{SI} \models u=v$ if and only if $\alpha(u)=\alpha(v)$. For a subset $B$ of an alphabet $A$ we denote by $[B]$ the set of all words over $A$ such that their content is $B$, i.e. $[B]=\left\{u \in A^{*} \mid \alpha(u)=B\right\}=\alpha^{-1}(B) \cap A^{*}$. In particular, for $B=\emptyset$ we write []$=[\emptyset]=\{\epsilon\}$ where $\epsilon$ is the empty word.

\subsection{Regular languages and syntactic quasiorder}

We recall the concept of syntactic quasiorder, which was introduced by Pin under the name syntactic order (see e.g. 13]).

For an arbitrary language $L \subseteq A^{*}$ we define relation $\leq_{L}$ on $A^{*}$ in the following way. For $u, v \in A^{*}$ we write $u \leq_{L} v$ if

$$
\left(\forall x, y \in A^{*}\right)(x v y \in L \Longrightarrow x u y \in L) .
$$

The relation $\leq_{L}$ is a stable quasiorder on the monoid $A^{*}$ and it is called the syntactic quasiorder of $L$. In this paper we deal only with regular languages, for which $\leq_{L}$ has finite index. The reason is that for a regular language $L$ there are only finitely many languages of the form $x^{-1} L y^{-1}=\left\{u \in A^{*} \mid x u y \in L\right\}$. We denote $M_{L}=A^{*} / \leq_{L}$ which is a finite ordered monoid, called the ordered syntactic monoid of $L$. The natural projection $\phi_{L}: A^{*} \rightarrow M_{L}$ is called the syntactic morphism. Note that $\phi_{L}(L)$ is an ideal in $\left(M_{L}, \leq\right)$ and that $\phi_{L}^{-1}\left(\phi_{L}(L)\right)=L$.

Now we consider the extension of $\phi_{L}$ to the continuous morphism $\phi_{L}: F_{A} \rightarrow M_{L}$ of compact monoids. The relation on $F_{A}$ consisting of the pairs $(u, v)$ such that $\phi_{L}(u) \leq \phi_{L}(v)$ is still denoted by $\leq_{L}$. Note that it is a stable quasiorder on $F_{A}$. Thus if we speak about syntactic quasiorder and syntactic morphism we can consider these extensions to $F_{A}$.

Using the continuous morphism $\phi_{L}$ we see that the topological closure $\bar{L}$ of $L$ is clopen in $F_{A}$ and that $\phi_{L}^{-1}\left(\phi_{L}(L)\right)=\bar{L}$. An alternative definition of the relation $\leq_{L}$ is given by the next lemma, whose proof amounts to an easy exercise. 
Lemma 2.1 Let $L$ be a regular language over an alphabet $A$ and let $u, v \in F_{A}$ be pseudowords. Then the following statements are equivalent:

(i) $u \leq_{L} v$,

(ii) for all $x, y \in F_{A}$, xvy $\in \bar{L}$ implies $x u y \in \bar{L}$,

(iii) for all $x, y \in A^{*}$, xvy $\in \bar{L}$ implies $x u y \in \bar{L}$.

\subsection{Some known results on the Straubing-Thérien hierarchy}

We recall the characterizations of $\mathcal{V}_{3 / 2}$, the level $3 / 2$ of the Straubing-Thérien hierarchy. The first is implicitly contained in [15].

Proposition 2.2 ([13, Theorem 8.8]) A language over an alphabet $A$ is of level $3 / 2$ if and only if it is a finite union of languages of the form $A_{0}^{*} a_{1} A_{1}^{*} a_{2} \cdots a_{k} A_{k}^{*}$, where each $A_{i} \subseteq A$ and each $a_{j} \in A$.

Proposition 2.3 ([18, Theorem 8.7], [13, Theorem 8.9]) A language is of level $3 / 2$ if and only if its ordered syntactic monoid satisfies the pseudoidentity $u^{\omega} v u^{\omega} \leq u^{\omega}$ for all pseudowords $u, v$ over some finite alphabet satisfying $\alpha(u)=\alpha(v)$.

The following proposition from [3] gives new pseudoidentities for the pseudovariety $\mathrm{V}_{2}$. The proof is also recalled as it is quite easy.

Proposition 2.4 ([3, Proposition 2]) Let $u$ and $v$ be pseudowords such that $\mathrm{V}_{3 / 2} \models u \leq v$. Then $\mathrm{V}_{2} \models$ $u^{\omega}=u^{\omega} v u^{\omega}$.

Proof: Since $\mathcal{V}_{2}$ is the Boolean closure of $\mathcal{V}_{3 / 2}$, we have $\mathrm{V}_{2} \models x=y$ if and only if $\mathrm{V}_{3 / 2} \models x=y$, i.e. if and only if $\mathrm{V}_{3 / 2} \models x \leq y$ and $\mathrm{V}_{3 / 2} \models y \leq x$.

From the assumption $\mathrm{V}_{3 / 2} \models u \leq v$, we deduce that $\alpha(u)=\alpha(v)$ because $\mathrm{SI} \subseteq \mathrm{V}_{3 / 2}$. From Proposition 2.3, we obtain immediately $\mathrm{V}_{3 / 2} \models u^{\omega} v u^{\omega} \leq u^{\omega}$.

When we multiply $u \leq v$ by $u^{\omega}$ from both sides, we obtain $u^{\omega} u u^{\omega} \leq u^{\omega} v u^{\omega}$. Since $\mathrm{V}_{3 / 2} \models x^{\omega+1}=$ $x^{\omega}$, we conclude that $\mathrm{V}_{3 / 2} \models u^{\omega} \leq u^{\omega} v u^{\omega}$.

\section{Relations on monoids related to level $3 / 2$}

For each finite alphabet $A$ and natural number $m$ we denote $\mathcal{U}_{m}(A)$ the set of all languages over $A$ of the form

$$
\left[A_{0}\right] a_{1}\left[A_{1}\right] a_{2}\left[A_{2}\right] \cdots\left[A_{k-1}\right] a_{k}\left[A_{k}\right]
$$

where $k \leq m, a_{1}, \ldots, a_{k} \in A$ and $A_{0}, \ldots, A_{k} \subseteq A$. Since $A$ is finite, so is each of the sets $\mathcal{U}_{m}(A)$. We denote $\mathcal{U}(A)$ their union over all $m \geq 0$. The languages of level $3 / 2$ over an alphabet $A$ are exactly the finite unions of languages from $\mathcal{U}(A)$, because for each finite alphabet $B$ one can express the language $B^{*}$ as the finite union of all languages of the form $[C]$ with $C \subseteq B$, which in turn are of level 1 .

Now, we define a relation $\preceq_{m}^{A}$ on the free profinite monoid $F_{A}$ in the following way:

$$
u \preceq_{m}^{A} v \text { if, for all } L \in \mathcal{U}_{m}(A), v \in \bar{L} \text { implies } u \in \bar{L} .
$$


Note that if $u \preceq_{m}^{A} v$ for some $u, v \in F_{A}$ then the implication $v \in \bar{L} \Longrightarrow u \in \bar{L}$ is satisfied also for each language $L$ which is a finite union of finite intersections of languages from $\mathcal{U}_{m}(A)$. One can prove that such languages form a positive variety of languages. This claim is a special case of [14, Theorem 5.1] or alternatively of [9. Theorem 1]. One can also prove it directly by showing that each morphic preimage and also each derivative of a language from the class $\mathcal{U}_{m}(A)$ is a finite union of finite intersections of languages from $\mathcal{U}_{m}(A)$. This method is used in 邨 to prove that the polynomial closure of a variety of languages constitutes a positive variety, although the formula for morphic preimage [4, Lemma 2.2.2] is not correct.ii

Lemma 3.1 Let $A$ be an alphabet, $m$ be a number and $u, v \in F_{A}$ be pseudowords. Then

$$
u \preceq_{m}^{A} v \text { if and only if, for all } L \in \mathcal{U}_{m}(A), u \leq_{L} v .
$$

In other words $\preceq_{m}^{A}$ is the intersection of all relations $\leq_{L}$ for $L \in \mathcal{U}_{m}(A)$.

Proof: Let $u, v \in F_{A}$ be such that $u \preceq_{m}^{A} v$ and let $L \in \mathcal{U}_{m}(A)$. We want to show that $u \leq_{L} v$, which is equivalent to condition (iii) in Lemma 2.1. Let $x, y \in A^{*}$ be such that $x v y \in \bar{L}$ and we show that also $x u y \in \bar{L}$. Since the set $\bar{L}$ is open, we may consider a sequence of words $\left(v_{n}\right)_{n \in \mathbb{N}}$ converging to $v$ such that for each $n$ we have $x v_{n} y \in L$. Hence $v_{n} \in x^{-1} L y^{-1}$ and we see that $v \in \bar{K}$ for $K=x^{-1} L y^{-1}$. The language $K$ is a finite union of finite intersections of languages from $\mathcal{U}_{m}(A)$ and the implication $v \in \bar{K} \Rightarrow u \in \bar{K}$ follows from $u \preceq_{m}^{A} v$. Thus there is a sequence of words $\left(u_{n}\right)_{n \in \mathbb{N}}$ converging to $u$ such that $u_{n} \in K=x^{-1} L y^{-1}$ for each $n \in \mathbb{N}$. Hence $x u_{n} y \in L$ and we deduce that $x u y \in \bar{L}$.

On the other hand, for $u, v \in F_{A}$ and $L \in \mathcal{U}_{m}(A)$ we have

$$
\left(u \leq_{L} v \text { and } v \in \bar{L}\right) \text { implies } u \in \bar{L}
$$

because we can consider condition (iii) in Lemma 2.1 with $x$ and $y$ empty words. This property entails the implication " $\Leftarrow$ ".

Given $X \subseteq F_{A}$, write $u \preceq_{X}^{A} v$ if $u, v \in F_{A}$ and $v \in X \Rightarrow u \in X$. Then $\preceq_{X}^{A}$ is a quasiorder in which the elements of $X$ are minima and the remaining elements are maxima.

Lemma 3.2 The following hold for every natural number $m$.

(i) For each $A$ the relation $\preceq_{m}^{A}$ is a stable quasiorder on $F_{A}$.

(ii) For every continuous morphism $\psi: F_{A} \rightarrow F_{B}$ we have

$$
\text { for all } u, v \in F_{A}, u \preceq_{m}^{A} v \text { implies } \psi(u) \preceq_{m}^{B} \psi(v) \text {. }
$$

(iii) The equivalence relation determined by the quasiorder $\preceq_{m}^{A}$ has index at most $2^{\left|\mathcal{U}_{m}(A)\right|}$.

\footnotetext{
(i) The problem lies in the fact that the formula does not take into account the possibility of a letter covering several of the distinguished letters. For instance, the formula fails for the homomorphism $\varphi: a^{*} \rightarrow b^{*}$ defined by $\varphi(a)=b^{2}$, for which $\varphi^{-1}([] b[] b[])=\{a\}=[] a[]$, while the language given by Arfi's formula is empty, since at least one of the derivatives in the languages in the union is empty.
} 
Proof: By Lemma 3.1, the relation $\preceq_{m}^{A}$ is the intersection of all stable quasiorders $\leq_{L}$ with $L \in \mathcal{U}_{m}(A)$, and therefore it is itself a stable quasiorder, which proves (ip. On the other hand, since $\preceq_{m}^{A}$ is also the intersection of the quasiorders of the form $\preceq \frac{A}{L}$, each of which has index 2 , we obtain (iii).

(iii) Let $u, v \in F_{A}$ be such that $u \preceq_{m}^{A} v$, let $L \in \mathcal{U}_{m}(B)$, and suppose that $\psi(v) \in \bar{L}$. From the continuity of $\psi$, we obtain the equality $\psi^{-1}(\bar{L})=\overline{\psi^{-1}(L)}$. Now $K=\psi^{-1}(L) \subseteq A^{*}$ is a finite union of finite intersections of languages from $\mathcal{U}_{m}(A)$. Since $v \in \bar{K}$ and $u \preceq_{m}^{A} v$ we have $u \in \bar{K}$. Hence $\psi(u) \in \bar{L}$, which proves that $\psi(u) \preceq_{m}^{B} \psi(v)$.

From Lemmas 3.1 and 3.2 one can also state that the ordered monoid $F_{A} / \preceq_{m}^{A}$ is a free (pro)finite ordered monoid in the pseudovariety of ordered monoids corresponding to the positive variety of languages generated by all $\mathcal{U}_{m}(B)$.

By a system of relations $\rho$ we mean an operator which determines, for each finite alphabet $A$, a relation $\rho^{A}$ on the free profinite monoid $F_{A}$. E.g. for each $m$ we have a system of relations $\preceq_{m}$. We call a system of relations $\rho$ a fully invariant system of stable quasiorders if it satisfies conditions (ii) and (iii) of Lemma 3.2. If every relation $\rho^{A}$ has finite index in $F_{A}$, then we speak of a fully invariant system of stable quasiorders of finite index.

Another example of a fully invariant system of stable quasiorders of finite index is given by the kernel of the content function $\alpha$. More precisely we consider the relation $\equiv^{A}$ on $F_{A}$ given by the following rule for $u, v \in F_{A}$ :

$$
u \equiv^{A} v \text { if } \alpha(u)=\alpha(v)
$$

Further, we define a relation $\preceq^{A}$ on $F_{A}$ as the intersection of all relations $\preceq_{m}^{A}$, i.e.

$$
u \preceq^{A} v \text { if, for all } L \in \mathcal{U}(A), v \in \bar{L} \text { implies } u \in \bar{L} .
$$

The following is an immediate consequence of Lemmas 3.1 and 3.2

Proposition 3.3 The system of relations $\preceq$ is a fully invariant system of stable quasiorders. For each alphabet $A$, the relation $\preceq^{A}$ is the intersection of all relations $\leq_{L}$ for $L \in \mathcal{U}(A)$.

Let $\varphi: F_{A} \rightarrow M$ be a continuous morphism to a finite monoid $M$. For a binary relation $R$ on $F_{A}$, we define the relation $\varphi(R)$ on the monoid $M$ by the rule

$$
\varphi(R)=\left\{(s, t) \in M \times M \mid \text { there exist } u, v \in F_{A} \text { such that } u R v, \varphi(u)=s \text { and } \varphi(v)=t\right\} .
$$

Note that if $R$ is a stable relation on $F_{A}$ then $\varphi(R)$ is a stable relation on $M$. But $\varphi(R)$ need not be a quasiorder even if $R$ is. We show that the definition of $\varphi(R)$ does not depend on $\varphi$ or $A$ when we consider a fully invariant system of stable quasiorders.

Lemma 3.4 Let $\varphi: F_{A} \rightarrow M$ and $\beta: F_{B} \rightarrow M$ be a pair of continuous morphisms. Further, let $\rho$ be $a$ fully invariant system of stable quasiorders.

(i) If $\beta$ is onto then $\varphi\left(\rho^{A}\right) \subseteq \beta\left(\rho^{B}\right)$.

(ii) If both $\varphi$ and $\beta$ are onto then $\varphi\left(\rho^{A}\right)=\beta\left(\rho^{B}\right)$. 
Proof: The second statement follows from applying the first one twice. So, assume that $\beta$ is onto and let $(s, t) \in \varphi\left(\rho^{A}\right)$. Then by the definition of $\varphi\left(\rho^{A}\right)$ we have $u, v \in F_{A}$ such that $(u, v) \in \rho^{A}, \varphi(u)=s$, and $\varphi(v)=t$. Since $\beta$ is onto and $B^{*}$ is dense in $F_{B}$, we can consider for each $a \in A$ a word $u_{a} \in B^{*}$ such that $\beta\left(u_{a}\right)=\varphi(a) \in M$. Then there is a continuous morphism $\psi: F_{A} \rightarrow F_{B}$ such that $\psi(a)=u_{a}$ for all $a \in A$, so that $\varphi=\beta \circ \psi$. Since $\rho$ is fully invariant we obtain $(\psi(u), \psi(v)) \in \rho^{B}$ from $(u, v) \in \rho^{A}$. From the definition of $\beta\left(\rho^{B}\right)$ we get

$$
(s, t)=(\varphi(u), \varphi(v))=(\beta(\psi(u)), \beta(\psi(v))) \in \beta\left(\rho^{B}\right),
$$

which completes the proof of the lemma.

Thus, we can define for each fully invariant system of stable quasiorders $\rho$ and every finite monoid $M$ the relation $\rho^{M}$ on $M$ by taking an arbitrary onto continuous morphism $\varphi: F_{A} \rightarrow M$ and putting $\rho^{M}=\varphi\left(\rho^{A}\right)$. In particular, we can consider the relations $\preceq^{M}$ and $\preceq_{m}^{M}$ for each $m$. The relation $\equiv^{M}$ is sometimes known in the literature as the set of SI-pointlike pairs of elements of $M$ (cf. [21, Subsection 2.4.1]).

We say that we have an effective description of a finite ordered monoid if its multiplication table and its order relation are known.

Useful properties of the relations $\preceq_{m}^{M}$ and $\preceq^{M}$ are given in the following lemma. Some of them are formulated in a more general setting.

Lemma 3.5 Let $\varphi: F_{A} \rightarrow M$ be an arbitrary onto continuous morphism and let $\rho$ be a fully invariant system of stable quasiorders; in particular $\rho^{M}=\varphi\left(\rho^{A}\right)$. Then the following properties hold.

(i) The relation $\rho^{M}$ is stable.

(ii) If the equivalence relation corresponding to $\rho^{A}$ has finite index then we have

$$
\rho^{M}=\left\{(s, t) \in M \times M \mid \text { there exist } u, v \in A^{*} \text { such that } u \rho^{A} v, \varphi(u)=s \text { and } \varphi(v)=t\right\} .
$$

Moreover, if we have an effective description of the finite ordered monoid $F_{A} / \rho^{A}$ then $\rho^{M}$ is computable.

(iii) For each $m$ we have $\preceq_{m+1}^{M} \subseteq \preceq_{m}^{M}$ and the relation $\preceq^{M}$ is the intersection of all relations $\preceq_{m}^{M}$.

Proof: (17) We already mentioned that $\varphi(R)$ is a stable relation whenever $R$ is a stable relation.

(iii) The inclusion " $\supseteq$ " is trivial. If we take $(s, t) \in \rho^{M}$ then we have $(u, v) \in \rho^{A}$ for some $u, v \in F_{A}$ such that $\varphi(u)=s$ and $\varphi(v)=t$. We denote $\eta$ the natural morphism from $F_{A}$ to the finite ordered monoid $F_{A} / \rho^{A}$. Since $\varphi^{-1}(s)$ and $\eta^{-1}(\eta(u))$ are both clopen subsets in $F_{A}$ which contain $u \in F_{A}$, it follows that their intersection is also non-empty and clopen in $F_{A}$. This entails that it contains some word $u^{\prime} \in A^{*}$. Hence we have $\varphi\left(u^{\prime}\right)=s$ and $\left(u^{\prime}, u\right) \in \rho^{A}$ (and also $\left(u, u^{\prime}\right) \in \rho^{A}$ ). By the same argument we obtain $v^{\prime} \in A^{*}$ such that $\varphi\left(v^{\prime}\right)=t$ and $\left(v, v^{\prime}\right) \in \rho^{A}$. Since $\rho^{A}$ is a transitive relation we get $\left(u^{\prime}, v^{\prime}\right) \in \rho^{A}$. We proved the first part of (iii).

For the second part we define a relational morphism $\pi$ from $M$ to $F_{A} / \rho^{A}$ as $\pi=\eta \circ \varphi^{-1}$. In other words $\pi=\left\{(\varphi(u), \eta(u)) \mid u \in A^{*}\right\}$. In fact $\pi$ is a submonoid of the finite monoid $M \times F_{A} / \rho^{A}$ and it is generated by the set $G=\{(\varphi(a), \eta(a)) \mid a \in A\}$. 
Since we have an effective description of the finite ordered monoid $F_{A} / \rho^{A}$ we can compute $\pi$ as the submonoid of the finite monoid $M \times F_{A} / \rho^{A}$ generated by $G$. Now $(s, t) \in \rho^{M}$ if and only if there exist $u_{\rho}, v_{\rho} \in F_{A} / \rho^{A}$ such that $u_{\rho} \leq v_{\rho}$ and $\left(s, u_{\rho}\right) \in \pi$ and $\left(t, v_{\rho}\right) \in \pi$. By the above, the latter condition on the pair $(s, t)$ can be effectively checked.

(iii) From the inclusions $\preceq^{A} \subseteq \preceq_{m+1}^{A} \subseteq \preceq_{m}^{A}$ it follows that $\preceq^{M} \subseteq \preceq_{m+1}^{M} \subseteq \preceq_{m}^{M}$. It remains to show that $\bigcap_{m \geq 0} \preceq_{m}^{M} \subseteq \preceq^{M}$. So, suppose that $s, t \in M$ are such that $s \preceq_{m}^{M} t$ for all $m \geq 0$. Then, for each $m \geq 0$ there exist $u_{m} \in \varphi^{-1}(s)$ and $v_{m} \in \varphi^{-1}(t)$ such that $u_{m} \preceq_{m}^{A} v_{m}$. Since the space $F_{A}$ is compact, there is a sequence of indices $m_{1}<m_{2}<\cdots$ such that each of the subsequences $\left(u_{m_{k}}\right)_{k}$ and $\left(v_{m_{k}}\right)_{k}$ converges to the respective limit $u$ and $v$. As $\varphi$ is continuous, we have $\varphi(u)=s$ and $\varphi(v)=t$. We claim that $u \preceq^{A} v$, which will establish that $s \preceq^{M} t$, as required. Indeed, given a language $L \in \mathcal{U}_{m}(A)$, consider the syntactic morphism $\phi_{L}: F_{A} \rightarrow M_{L}$, which is continuous. Hence there is some $k$ such that $m_{k} \geq m$ and $\phi_{L}\left(u_{m_{k}}\right)=\phi_{L}(u)$ and $\phi_{L}\left(v_{m_{k}}\right)=\phi_{L}(v)$. Since $u_{m_{k}} \preceq_{m_{k}}^{A} v_{m_{k}}$ and $L \in \mathcal{U}_{m}(A)$, we conclude in particular that $u_{m_{k}} \leq_{L} v_{m_{k}}$, whence also $u \leq_{L} v$, which proves the claim.

Recall that we have defined the relation $\tau_{3 / 2}(M) \subseteq M \times M$ by the rule $(s, t) \in \tau_{3 / 2}(M)$ if there are a finite set of variables $X$, a pair of pseudowords $u, v \in F_{X}$ such that $\mathrm{V}_{3 / 2} \models u \leq v$, and a continuous morphism $\varphi: F_{X} \rightarrow M$ such that $\varphi(u)=s, \varphi(v)=t$. We show that the inequalities satisfied in $\mathrm{V}_{3 / 2}$ are exactly given by the system of relations $\preceq$ and that the relation $\tau_{3 / 2}(M)$ coincides with the previous relation $\preceq^{M}$.

Proposition 3.6 Let $A$ be a finite alphabet.

(i) For each $u, v \in F_{A}$ we have

$$
\mathrm{V}_{3 / 2} \models u \leq v \text { if and only if } u \preceq^{A} v \text {. }
$$

(ii) For every finite monoid $M$ we have $\tau_{3 / 2}(M)=\preceq^{M}$.

Proof: (i) Let $u, v \in F_{A}$ be such that $\mathrm{V}_{3 / 2} \models u \leq v$. Then for each $L \in \mathcal{U}(A)$ and its syntactic morphism $\phi_{L}: F_{A} \rightarrow M_{L}$ we have $\phi_{L}(u) \leq \phi_{L}(v)$, i.e. $u \leq_{L} v$. This shows that $u \preceq^{A} v$ by Proposition 3.3.

Now, let $u \preceq^{A} v$. We want to show that for each $L \in \mathcal{V}_{3 / 2}(B)$ and its syntactic morphism $\phi_{L}: F_{B} \rightarrow$ $M_{L}$ onto the ordered syntactic monoid $M_{L}$ of $L$, we have $M_{L} \models u \leq v$. It is enough to prove it for languages $L \in \mathcal{U}(B)$ because $\mathcal{V}_{3 / 2}$ is the positive variety of languages generated by all $\mathcal{U}(C)$. So, let $L \in \mathcal{U}(B)$ with syntactic morphism $\phi_{L}: F_{B} \rightarrow M_{L}$ and take $\psi: F_{A} \rightarrow M_{L}$ an arbitrary continuous morphism. Since the syntactic morphism $\phi_{L}$ is onto we obtain $\psi\left(\preceq^{A}\right) \subseteq \phi_{L}\left(\preceq^{B}\right)$ from Lemma 3.4. Thus, as we are assuming that $u \preceq^{A} v$, so that $(\psi(u), \psi(v)) \in \bar{\psi}\left(\preceq^{A}\right)$, we conclude that there are $u^{\prime}, v^{\prime} \in F_{B}$ such that $\psi(u)=\phi_{L}\left(u^{\prime}\right), \psi(v)=\phi_{L}\left(v^{\prime}\right)$, and $u^{\prime} \preceq^{B} v^{\prime}$. Proposition 3.3 yields $u^{\prime} \leq_{L} v^{\prime}$ and we get $\psi(u)=\phi_{L}\left(u^{\prime}\right) \leq_{L} \phi_{L}\left(v^{\prime}\right)=\psi(v)$ in $M_{L}$. We have thus proved that $M_{L} \models u \leq v$.

(iii) We fix the finite alphabet $B=M$ and onto continuous morphism $\beta: F_{B} \rightarrow M$ given by $\left.\beta\right|_{B}=$ $\operatorname{id}_{B}$ and we show that $\tau_{3 / 2}(M)=\beta\left(\preceq^{B}\right)$. The inclusion $\beta\left(\preceq^{B}\right) \subseteq \tau_{3 / 2}(M)$ follows from (1i). Let $(s, t) \in \tau_{3 / 2}(M)$. Then there are a finite set of variables $X$, a pair of pseudowords $u, v \in F_{X}$ such that $\mathrm{V}_{3 / 2} \models u \leq v$ and a continuous morphism $\varphi: F_{X} \rightarrow M$ such that $\varphi(u)=s, \varphi(v)=t$. Hence we have $u \preceq^{X} v$ from (i). Lemma 3.4 then yields $(s, t) \in \varphi\left(\preceq^{X}\right) \subseteq \beta\left(\preceq^{B}\right)$. 


\section{New upper bound}

In the first section we have defined the pseudovariety of finite monoids

$$
\mathrm{F}=\llbracket u^{\omega}=u^{\omega} v u^{\omega} \mid \mathrm{V}_{3 / 2} \models u \leq v \rrbracket .
$$

Our goal is to solve the membership problem for F. So, for a fixed monoid $M$ we need to test whether

$$
s^{\omega}=s^{\omega} t s^{\omega}
$$

for all $(s, t) \in \tau_{3 / 2}(M)=\preceq^{M}$. The crucial trick in our contribution is the following lemma.

Lemma 4.1 Let $R$ be a reflexive stable relation on a given finite monoid $M$. Then condition (4.1) holds for every pair $(s, t)$ from $R$ if and only if condition (4.1) holds for every pair $(s, t)$ from the transitive closure of $R$.

Proof: Assume that condition (4.1) holds for every pair $(s, t)$ from $R$. We show by induction with respect to $i$ that condition (4.1) holds for every pair $(s, t)$ from $R^{i}$. For $i=1$, this is given. Let $i>1$ and $(s, t) \in R^{i}$ be an arbitrary pair. Thus there is $z \in M$ such that $(s, z) \in R^{i-1}$ and $(z, t) \in R$. Since $R$ is a stable relation we have $\left(s^{\omega} z s^{\omega}, s^{\omega} t s^{\omega}\right) \in R$. Condition 4.1 holds for $R$, hence we have

$$
\left(s^{\omega} z s^{\omega}\right)^{\omega}=\left(s^{\omega} z s^{\omega}\right)^{\omega}\left(s^{\omega} t s^{\omega}\right)\left(s^{\omega} z s^{\omega}\right)^{\omega} .
$$

On the other hand, by the induction hypothesis, since $(s, z) \in R^{i-1}$, we have $s^{\omega}=s^{\omega} z s^{\omega}$. Hence $s^{\omega}=\left(s^{\omega} z s^{\omega}\right)^{\omega}$ and we get

$$
s^{\omega}=\left(s^{\omega} z s^{\omega}\right)^{\omega}=\left(s^{\omega} z s^{\omega}\right)^{\omega}\left(s^{\omega} t s^{\omega}\right)\left(s^{\omega} z s^{\omega}\right)^{\omega}=s^{\omega}\left(s^{\omega} t s^{\omega}\right) s^{\omega}=s^{\omega} t s^{\omega} .
$$

Now since condition 4.1) holds for every pair $(s, t)$ from $R^{i}$ for every $i$, condition (4.1) holds for all pairs $(s, t)$ from $R^{*}=\bigcup_{i \in \mathbb{N}} R^{i}$. The opposite implication in the statement is trivial, because $R \subseteq R^{*}$.

If we apply this observation to our relation $\preceq^{M}$ then we obtain the following basic characterization of the pseudovariety $\mathrm{F}$.

Proposition 4.2 Let $M$ be a finite monoid. Then $M \in \mathrm{F}$ if and only if the condition (4.1) holds for every pair $(s, t)$ from the transitive closure of the relation $\preceq^{M}$.

Proof: The statement is an easy consequence of the definition of the class F, and Proposition 3.6 and Lemma 4.1.

We should show that the transitive closure of $\preceq^{M}$ can be computed. In fact, we give an alternative characterization of this relation which is motivated by Proposition 2.3. We define

$$
S_{M}=\{(s, s) \in M \times M \mid s \in M\} \cup\left\{\left(s^{\omega} t s^{\omega}, s^{\omega}\right) \in M \times M \mid s \equiv^{M} t\right\} .
$$

Furthermore, we denote by $\left\langle S_{M}\right\rangle$ the submonoid of the monoid $M \times M$ generated by the set $S_{M}$ and we denote the transitive closure of the relation $\left\langle S_{M}\right\rangle$, i.e. $\left\langle S_{M}\right\rangle^{*}$, by $T_{M}$. The two following results clarify the meaning of $T_{M}$. 
Lemma 4.3 For each finite monoid $M$ the relation $T_{M}$ is a computable stable quasiorder.

Proof: Since $M$ is finite and $\equiv$ is a fully invariant system of stable quasiorders of finite index, the relation $\equiv^{M}$ is computable by Lemma 3.5. Hence one can compute also the relation $S_{M}$. Generating the submonoid $\left\langle S_{M}\right\rangle$ and then the transitive closure $\left\langle S_{M}\right\rangle^{*}=T_{M}$ is routine. The fact that $T_{M}$ is stable follows from a general observation concerning the transitive closure of a stable relation.

Proposition 4.4 Let $M$ be a finite monoid. Then the transitive closure of $\preceq^{M}$ is $T_{M}$.

Proof: We have $S_{M} \subseteq \preceq^{M}$ by Proposition 2.3 and Proposition 3.6 (ii). By Lemma 3.5 (月), we have $\left\langle S_{M}\right\rangle \subseteq \preceq^{M}$. Thus the transitive closure of $\left\langle S_{M}\right\rangle$, i.e. $T_{M}$, is a subset of the transitive closure of $\preceq^{M}$. Thus we proved that $T_{M} \subseteq\left(\preceq^{M}\right)^{*}$. The reverse inclusion $\left(\preceq^{M}\right)^{*} \subseteq T_{M}$ is much more difficult. By Lemma 3.5 (iii) we know that $\preceq^{M} \subseteq \preceq_{m}^{M}$ for every natural number $m$. Hence we have $\left(\preceq^{M}\right)^{*} \subseteq\left(\preceq_{m}^{M}\right)^{*}$ for every $m$. To prove $\left(\preceq^{M}\right)^{*} \subseteq T_{M}$ it is therefore enough to prove that there is a number $m$ with the property $\left(\preceq_{m}^{M}\right)^{*} \subseteq T_{M}$. This statement is contained in Proposition 5.2 in the next section.

\section{Application of the Factorization Forest Theorem}

To finish the proof of Proposition 4.4 we use the Factorization Forest Theorem of Imre Simon. In fact, our proof is inspired by the proof concerning polynomial closures given by Pin and Weil [18] (see also [5, Section 3]). The following brief introduction to factorization forests is essentially borrowed from [18, Section 4].

A factorization forest is a mapping from $A^{2} A^{*}$ to $\bigcup_{n \geq 2}\left(A^{+}\right)^{n}$ which associates to every word $x \in A^{*}$ of length at least 2 a factorization $d(x)=\left(x_{1}, x_{2}, \ldots, x_{n}\right)$ of $x$ such that $n \geq 2, x_{1}, x_{2}, \ldots, x_{n} \in A^{+}$ and $x_{1} x_{2} \cdots x_{n}=x$. The integer $n$ is called the degree of the factorization $d(x)$. Thus a factorization forest is a description of a recursive process to factorize words as products of letters. The height function of a factorization forest $d$ is a mapping $h_{d}: A^{+} \rightarrow \mathbb{N}$ defined by the rule

$$
h_{d}(x)= \begin{cases}0 & \text { if } x \in A \\ 1+\max \left\{h_{d}\left(x_{i}\right) \mid 1 \leq i \leq n\right\} & \text { if } d(x)=\left(x_{1}, x_{2}, \ldots, x_{n}\right) .\end{cases}
$$

The height of a word $x$ in a factorization forest $d$ is simply $h_{d}(x)$ and the height of $d$ is given by $H_{d}=$ $\sup \left\{h_{d}(x) \mid x \in A^{+}\right\}$.

For a given word $x \in A^{+}$, we define recursively the so-called factorization tree of $x$ as a tree with root $x$ and direct descendants $x_{1}, x_{2}, \ldots, x_{n}$, where $d(x)=\left(x_{1}, x_{2}, \ldots, x_{n}\right)$, and the subtree with root $x_{i}$ being the factorization tree of $x_{i}$.

Let $M$ be a finite semigroup and let $\varphi: A^{+} \rightarrow M$ be a morphism. A factorization forest $d$ is Ramseyan modulo $\varphi$ if, for every word $x \in A^{2} A^{*}$, either $d(x)$ is of degree 2 or there exists an idempotent $e \in M$ such that $d(x)=\left(x_{1}, x_{2}, \ldots, x_{n}\right)$ with $n \geq 3$ and $\varphi\left(x_{1}\right)=\varphi\left(x_{2}\right)=\cdots=\varphi\left(x_{n}\right)=e$.

These definitions apply in particular if the semigroup $M$ is a monoid. Because there is no interest in factorizations of the empty word, when we speak about a factorization forest modulo $\varphi: A^{*} \rightarrow M$ we mean a factorization forest modulo $\left.\varphi\right|_{A^{+}}: A^{+} \rightarrow M$.

The Factorization Forest Theorem states that for every morphism $\varphi$ to a given finite semigroup $M$ there exists a factorization forest of finite height which is Ramseyan modulo $\varphi$. Originally the theorem 
was proved by I. Simon with the upper bound $9|M|$ in [23] and with an exponential upper bound but with easier proof in [24]. In successive papers the upper bound has been improved (see e.g. [10] for references). The most recent paper on the topic [10] establishes the upper bound $3|M|-1$ in the general case and the upper bound $2|M|$ in the case of aperiodic semigroups, which are both optimal for the respective classes of semigroups.

In our application the exact upper bound does not play any role, we use just the existence of it. So we may as well formulate the theorem in existential form.

Theorem 5.1 ([23, 24]) Let $M$ be a finite monoid and $\varphi: A^{*} \rightarrow M$ be a morphism. Then there exists a factorization forest of finite height which is Ramseyan modulo $\varphi$.

Now we can return to our considerations. The missing part of the proof of Proposition 4.4 is the following statement. Note that, for our purposes, it is irrelevant whether an integer $m$ as in the statement of Proposition 5.2 is computable, although from the proof below and the Factorization Forest Theorem it follows that such an integer can be indeed be computed.

Proposition 5.2 Let $M$ be a finite monoid and $\varphi: A^{*} \rightarrow M$ be a morphism. Then there exists an integer $m$ such that $\left(\preceq_{m}^{M}\right)^{*} \subseteq T_{M}$.

Proof: Let $d$ be a factorization forest of finite height which is Ramseyan modulo $\varphi$, whose existence is ensured by Theorem 5.1. For the purpose of this proof, we could choose any strict upper bound $K$ of the height $H_{d}$ of $d$. For definiteness, we put $K=H_{d}+1$. We prove that the inclusion in the statement holds for $m=2^{K+1}$. Since $T_{M}$ is a transitive relation it is enough to establish that $\preceq_{m}^{M} \subseteq T_{M}$.

Let $s, t \in M$ and suppose that $(s, t) \in \preceq_{m}^{M}$. By Lemma 3.5 (iii) we can take $u, v \in A^{*}$ such that $\varphi(u)=s, \varphi(v)=t$ and $u \preceq_{m}^{A} v$.

Intuitively, we take a factorization tree of $v$, and in every factorization used in this tree, i.e. $d(x)=$ $\left(x_{1}, x_{2}, \ldots, x_{n}\right)$ we keep just the first and last factors, i.e. $x_{1}$ and $x_{n}$, which we further factorize and instead of the middle part $\left(x_{2}, \ldots, x_{n-1}\right)$ we take just the product $x_{2} \cdots x_{n-1}$ which we do not factorize any further. Thus at the end of the process we obtain a factorization of the word $v$ of the form $v=$ $a_{1} v_{1} a_{2} v_{2} \cdots v_{k-1} a_{k}$ where $a_{i} \in A$ and $v_{i} \in A^{*}$. Moreover, since $h_{d}(v)<K$ we have $k<2^{K+1}=m$. We are led to consider the language

$$
L=[] a_{1}\left[\alpha\left(v_{1}\right)\right] a_{2} \cdots a_{k-1}\left[\alpha\left(v_{k-1}\right)\right] a_{k}[] \in \mathcal{U}_{m}(A) .
$$

Since $v \in L$ and $u \preceq_{m}^{A} v$ we have $u \in L$. Thus we can write $u=a_{1} u_{1} a_{2} u_{2} \cdots a_{k-1} u_{k-1} a_{k}$ where the $u_{i} \in A^{*}$ are such that $\alpha\left(u_{i}\right)=\alpha\left(v_{i}\right)$ for every $i=1, \ldots, k-1$.

Now, if we consecutively replace $v_{i}$ for $u_{i}$ in the mentioned factorization of $v$, where the order of replacements is given by the structure of the factorization tree of $v$, then the images under $\phi$ of the corresponding pairs of words in this sequence are in the relation $T_{M}$. Thus at the end of the process we obtain $(s, t)=(\phi(u), \phi(v)) \in T_{M}$. This is just the underlying idea of the proof which we now proceed to make more precise.

First, we consider a full binary tree of height $K$, i.e. $\mathbb{T}=\bigcup_{i=0}^{K}\{1,2\}^{i}=\left\{p \in\{1,2\}^{*}|| p \mid \leq K\right\}$, where each node $p \in \mathbb{T}$ which is not a leaf, i.e. $|p|<K$, has left child $p 1$ and right child $p 2$. The root of $\mathbb{T}$, i.e. the empty word over the alphabet $\{1,2\}$, is denoted by $\lambda$. For technical reasons, we want to distinguish this empty word from the empty word over the alphabet $A$, which is denoted $\epsilon$. In fact the 
tree structure of $\mathbb{T}$ is not needed for the following proof, but it can help to follow it. We mention that $|\mathbb{T}|=\sum_{i=0}^{K} 2^{i}=2^{K+1}-1=m-1$.

Now, for our propose, we define a certain order, denoted by $\sqsubseteq$, on the set $\mathbb{T}$ which is known in the literature under the names "in-order traversal" and "symmetric traversal" of the tree. For a pair of nodes $p, q \in \mathbb{T}$ we denote $\langle p, q\rangle \in \mathbb{T}$ the longest common prefix of $p$ and $q$ and we put $p \sqsubseteq q$ if one of the following conditions is satisfied:

- $p=\langle p, q\rangle 1 p^{\prime}$ and $q=\langle p, q\rangle 2 q^{\prime}$ for some $p^{\prime}, q^{\prime} \in \mathbb{T}$,

- $p=\langle p, q\rangle 1 p^{\prime}$ and $q=\langle p, q\rangle$ for some $p^{\prime} \in \mathbb{T}$,

- $p=\langle p, q\rangle$ and $q=\langle p, q\rangle 2 q^{\prime}$ for some $q^{\prime} \in \mathbb{T}$,

- $p=\langle p, q\rangle=q$.

We write $p \sqsubset q$ if $p \sqsubseteq q$ and $p \neq q$. Taking arbitrary nodes $p, q \in \mathbb{T}$, such that $p \neq q$, exactly one of the possibilities $p \sqsubset q$ and $q \sqsubset p$ occurs. Elementary computations check that $\sqsubseteq$ is also transitive. Altogether, the relation $\sqsubseteq$ is a linear ordering of $\mathbb{T}$.

We say that a pair $(\chi, \bar{\chi})$ is a compatible labeling on $\mathbb{T}$ if the following conditions are satisfied:

(i) both $\chi: \mathbb{T} \rightarrow A^{*}$ and $\bar{\chi}: \mathbb{T} \rightarrow A^{*}$ are mappings;

(ii) for each leaf $p \in \mathbb{T}$, i.e. $p$ such that $|p|=K$, we have $\bar{\chi}(p)=\chi(p)$;

(iii) for each $p \in \mathbb{T}$ which is not a leaf, i.e. $|p|<K$, we have $\bar{\chi}(p)=\bar{\chi}(p 1) \cdot \chi(p) \cdot \bar{\chi}(p 2)$.

We call the word $\bar{\chi}(\lambda) \in A^{*}$ the value of the compatible labeling on $\mathbb{T}$ and we also denote it $\bar{\chi}(\mathbb{T})$.

Note that, for an arbitrary compatible labeling $\chi$ on $\mathbb{T}$, we have

$$
\bar{\chi}(\lambda)=\chi\left(p_{1}\right) \chi\left(p_{2}\right) \cdots \chi\left(p_{m-1}\right)
$$

where $p_{1} \sqsubset p_{2} \sqsubset \cdots \sqsubset p_{m-1}$ are all nodes from $\mathbb{T}$. Indeed, one can prove by induction with respect to the tree $\mathbb{T}$ in bottom-up direction, that for each $p \in \mathbb{T}$ we have $\bar{\chi}(p)=\chi\left(p p_{1}\right) \cdots \chi\left(p p_{i}\right)$ where $p p_{1} \sqsubset \cdots \sqsubset p p_{i}$ are all nodes from $\mathbb{T}$ with the prefix $p$. Thus, if $\chi: \mathbb{T} \rightarrow A^{*}$ is an arbitrary mapping, then there is a unique mapping $\bar{\chi}$ such that the pair $(\chi, \bar{\chi})$ forms a compatible labeling on $\mathbb{T}$. For this reason a compatible labeling $(\chi, \bar{\chi})$ is usually referred simply by $\chi$.

We say that a labeling $\xi$ on $\mathbb{T}$ is similar to a labeling $\chi$ on $\mathbb{T}$ if the following conditions are satisfied:

(i) for each $p \in \mathbb{T}$ we have $\alpha(\xi(p))=\alpha(\chi(p))$;

(ii) for each $p \in \mathbb{T}$ such that $\chi(p) \in A$ we have $\xi(p)=\chi(p)$.

Note that this relation is not symmetric, because for some $p \in \mathbb{T}$ and $a \in A$ we can have $\chi(p)=a a a$ and $\xi(p)=a$ and in this case $\chi$ is not similar to $\xi$ but $\xi$ could be similar to $\chi$.

For the word $v$ we will define a certain compatible labeling $\left(\chi_{v}, \bar{\chi}_{v}\right)$ on $\mathbb{T}$. The definition is inductive in top-down direction with respect to the tree $\mathbb{T}$ and it reflects the factorization of $v$ in the factorization forest $d$. First of all, we put $\bar{\chi}_{v}(\lambda)=v$. Next, if for $p \in \mathbb{T}$ which is not a leaf, i.e. $|p|<K$, we have defined $\bar{\chi}_{v}(p)=x \in A^{*}$, then we define the values $\chi_{v}(p), \bar{\chi}_{v}(p 1)$ and $\bar{\chi}_{v}(p 2)$ as follows: 
(a) if $x=\epsilon$ then we put $\bar{\chi}_{v}(p 1)=\chi_{v}(p)=\bar{\chi}_{v}(p 2)=\epsilon$;

(b) if $x \in A$ then we put $\bar{\chi}_{v}(p 1)=\epsilon, \chi_{v}(p)=x$ and $\bar{\chi}_{v}(p 2)=\epsilon$;

(c) if $d(x)=\left(x_{1}, x_{2}\right)$ then we put $\bar{\chi}_{v}(p 1)=x_{1}, \chi_{v}(p)=\epsilon$ and $\bar{\chi}_{v}(p 2)=x_{2}$;

(d) if $d(x)=\left(x_{1}, x_{2}, \ldots, x_{n}\right), n>2$ then we put $\bar{\chi}_{v}(p 1)=x_{1}, \chi_{v}(p)=x_{2} x_{3} \cdots x_{n-1}$ and $\bar{\chi}_{v}(p 2)=$ $x_{n}$.

Finally, if for a leaf $p \in \mathbb{T}$ we have defined $\bar{\chi}_{v}(p)=x \in A^{*}$, then we put $\chi_{v}(p)=x$. Since $h_{d}(v)<K$ we can see that in this case $\chi_{v}(p)=\bar{\chi}_{v}(p)=x=\epsilon$.

Directly from the definition of the pair $\left(\chi_{v}, \bar{\chi}_{v}\right)$ we see that it is a compatible labeling on $\mathbb{T}$ with the value $\bar{\chi}_{v}(\mathbb{T})=\bar{\chi}_{v}(\lambda)=v$.

We have defined all technical notation and we can formulate the crucial observations which finish the proof of the proposition.

Lemma 5.3 Let $v \in A^{*}$ be an arbitrary word and $\left(\chi_{v}, \bar{\chi}_{v}\right)$ be the compatible labeling on $\mathbb{T}$ given by the previous definition. Let $u \in A^{*}$ be such that $u \preceq_{m}^{A} v$. Then there is a compatible labeling $\xi$ on $\mathbb{T}$ which is similar to $\chi_{v}$ and which has the value $\bar{\xi}(\mathbb{T})=\bar{\xi}(\lambda)=u$.

Lemma 5.4 Let $v$ be an arbitrary word and $\left(\chi_{v}, \bar{\chi}_{v}\right)$ be the compatible labeling on $\mathbb{T}$ given by the previous definition. If $\xi$ is a compatible labeling on $\mathbb{T}$ similar to $\chi_{v}$ then

$$
\left(\varphi(\bar{\xi}(\mathbb{T})), \varphi\left(\bar{\chi}_{v}(\mathbb{T})\right)\right) \in T_{M}
$$

Proof of Lemma 5.3: Let $v \in A^{*}$ and consider the corresponding compatible labeling $\chi_{v}$ and $u \in A^{*}$ such that $u \preceq_{m}^{A} v$. We denote $\mathbb{S}=\left\{p \in \mathbb{T} \mid \chi_{v}(p) \neq \epsilon\right\}$. Then for each $p \in \mathbb{S}$ let $a_{p} \in A$ be the last letter in $\chi_{v}(p)$ and let $v_{p} \in A^{*}$ be such that $v_{p} a_{p}=\chi_{v}(p)$. Thus $v=v_{p_{1}} a_{p_{1}} v_{p_{2}} a_{p_{2}} \cdots v_{p_{k}} a_{p_{k}}$, where $k<m$ and $p_{1} \sqsubset p_{2} \sqsubset \cdots \sqsubset p_{k}$ are all nodes from $\mathbb{S}$. We denote $A_{i}=\alpha\left(v_{p_{i}}\right)$ for $i=1, \ldots, k$. We consider the language

$$
L=\left[A_{1}\right] a_{p_{1}}\left[A_{2}\right] a_{p_{2}} \cdots\left[A_{k}\right] a_{p_{k}}[] .
$$

As $v \in L \in \mathcal{U}_{m}(A)$ and $u \preceq_{m}^{A} v$, we deduce that $u \in L$ and we can write $u=u_{p_{1}} a_{p_{1}} u_{p_{2}} a_{p_{2}} \cdots u_{p_{k}} a_{p_{k}}$, where for each $i=1, \ldots, k$ we have $\alpha\left(u_{p_{i}}\right)=\alpha\left(v_{p_{i}}\right)=A_{i}$.

We define a compatible labeling $\xi$ on $\mathbb{T}$ in the following way: if $p \notin \mathbb{S}$, i.e. $\chi_{v}(p)=\epsilon$, then we put $\xi(p)=\epsilon$ and if $p \in \mathbb{S}$ then we put $\xi(p)=u_{p} a_{p}$. Now for each $p \in \mathbb{T}$ we have $\alpha(\xi(p))=\alpha\left(\chi_{v}(p)\right)$. Moreover, if $\chi_{v}(p) \in A$ then $\chi_{v}(p)=a_{p}$ and $v_{p}=\epsilon$, which entails $u_{p}=\epsilon$ and, consequently, $\xi(p)=$ $a_{p}=\chi_{v}(p)$. Thus the constructed compatible labeling $\xi$ on $\mathbb{T}$ is similar to $\chi_{v}$. We know that $\bar{\xi}(\lambda)$ is the product of all $\xi(p)$, for $p \in \mathbb{T}$, in the order $\sqsubset$. Since $\xi(p)=\epsilon$ for $p \notin \mathbb{S}$, this product is equal to $u_{p_{1}} a_{p_{1}} u_{p_{2}} a_{p_{2}} \cdots u_{p_{k}} a_{p_{k}}=u$ where $p_{1} \sqsubset p_{2} \sqsubset \cdots \sqsubset p_{k}$ are all nodes from $\mathbb{S}$.

Proof of Lemma 5.4: We prove the statement by an induction with respect to the structure of $\mathbb{T}$ in bottom-up direction. This means that we want to prove for each $p \in \mathbb{T}$ that

$$
\left(\varphi(\bar{\xi}(p)), \varphi\left(\bar{\chi}_{v}(p)\right)\right) \in T_{M} .
$$

First, if $p$ is a leaf in $\mathbb{T}$ then $\chi_{v}(p)=\bar{\chi}_{v}(p)=\epsilon$, because the height satisfies the inequality $h_{d}(v)<K$. Thus $\bar{\xi}(p)=\bar{\chi}(p)$ for this leaf $p$ and (5.1) is trivial. 
Now, let $p$ be not a leaf and assume that $(5.1)$ is true for the nodes $p 1$ and $p 2$. We distinguish between the cases (a)-(d) in the definition of $\chi_{v}$ which were used for the definition of $\chi_{v}(p)$.

In case (a), we have $\bar{\chi}_{v}(p)=\epsilon$ which entails $\chi_{v}(p q)=\epsilon$ for all $q \in \mathbb{T}$ such that $p q \in \mathbb{T}$. It follows that $\xi(p q)=\epsilon$ for all such $q \in \mathbb{T}$. Consequently $\bar{\xi}(p)=\epsilon$ and (5.1) is trivially satisfied.

In case (b), we have $\chi_{v}(p)=x \in A, \bar{\chi}_{v}(p 1)=\bar{\chi}_{v}(p 2)=\epsilon$. Since $\xi$ is similar to $\chi_{v}$ we have $\xi(p)=\chi_{v}(p)=x$ and $\bar{\xi}(p 1)=\bar{\xi}(p 2)=\epsilon$. Consequently $\bar{\xi}(p)=\bar{\chi}_{v}(p)=x$ and (5.1) is again trivially satisfied.

In case (c), we have $\chi_{v}(p)=\epsilon$ which implies $\xi(p)=\epsilon=\chi_{v}(p)$. Then $\bar{\xi}(p)=\bar{\xi}(p 1) \bar{\xi}(p 2)$ and $\bar{\chi}_{v}(p)=\bar{\chi}_{v}(p 1) \bar{\chi}_{v}(p 2)$. Since $\left(\varphi(\bar{\xi}(p 1)), \varphi\left(\bar{\chi}_{v}(p 1)\right)\right) \in T_{M}$ and $\left(\varphi(\bar{\xi}(p 2)), \varphi\left(\bar{\chi}_{v}(p 2)\right)\right) \in T_{M}$ we obtain (5.1) as a consequence of the fact that $T_{M}$ is a stable relation on $M$.

In case $(\mathrm{d})$, we have $\bar{\chi}_{v}(p 1)=x_{1}, \chi_{v}(p)=x_{2} \cdots x_{n-1}$ and $\bar{\chi}_{v}(p 2)=x_{n}$, and consequently $\bar{\chi}_{v}(p)=$ $x=x_{1} x_{2} \cdots x_{n}$. Moreover, $\varphi\left(x_{1}\right)=\varphi\left(x_{2}\right)=\cdots=\varphi\left(x_{n}\right)=e$ is an idempotent in $M$. Since $\xi$ is similar to $\chi_{v}$ we have $\xi(p)=w \in A^{*}$ such that $\alpha(w)=\alpha\left(x_{2} x_{3} \cdots x_{n-1}\right)$. This means that $\varphi(w) \equiv^{M} \varphi\left(x_{2} x_{3} \cdots x_{n-1}\right)=e$ and we have $(e \cdot \varphi(w) \cdot e, e) \in S_{M} \subseteq T_{M}$. By induction hypothesis we have $\left(\varphi(\bar{\xi}(p 1)), \varphi\left(\bar{\chi}_{v}(p 1)\right)\right) \in T_{M}$, where the second coordinate is $e$ because $\bar{\chi}_{v}(p 1)=x_{1}$ and $\varphi\left(x_{1}\right)=e$. Thus $(\varphi(\bar{\xi}(p 1)), e) \in T_{M}$ and, analogously, $(\varphi(\bar{\xi}(p 2)), e) \in T_{M}$. Since $T_{M}$ is a stable quasiorder we get the following sequence of $T_{M}$-related elements of $M$. (Here we write $T_{M}$ in an infix notation to make the presentation more readable.)

$$
\varphi(\bar{\xi}(p))=\varphi(\bar{\xi}(p 1)) \cdot \varphi(\xi(p)) \cdot \varphi(\bar{\xi}(p 2)) T_{M} e \cdot \varphi(w) \cdot e T_{M} e=\varphi\left(x_{1} x_{2} \cdots x_{n}\right)=\varphi\left(\bar{\chi}_{v}(p)\right) .
$$

This achieves the proof of Lemma 5.4 .

From Lemmas 5.3 and 5.4 it immediately follows that

$$
(s, t)=(\varphi(u), \varphi(v))=\left(\varphi(\bar{\xi}(\mathbb{T})), \varphi\left(\bar{\chi}_{v}(\mathbb{T})\right)\right) \in T_{M} .
$$

Thus we have proved the inclusion $\preceq_{m}^{M} \subseteq T_{M}$ and the proof of Proposition 5.2 is finished.

Combining Propositions 4.2, 4.4 and 5.2, we obtain the following characterization of our new upper bound F.

Theorem 5.5 The pseudovariety $\mathrm{F}$ consists of all finite monoids $M$ such that $s^{\omega}=s^{\omega} t s^{\omega}$ whenever $(s, t) \in T_{M}$.

Proof: By Proposition 4.4, which is a consequence of Proposition 5.2, the relation $T_{M}$ is equal to the transitive closure of $\preceq^{M}$. Hence we get the statement by applying Proposition 4.2 .

From Theorem 5.5, we obtain the following main result of this paper.

Theorem 5.6 It is decidable whether a given finite monoid $M$ belongs to the pseudovariety $\mathrm{F}$.

Proof: It suffices to observe that the necessary and sufficient condition on a finite monoid $M$ to belong to $\mathrm{F}$ given by Theorem 5.5 can be effectively checked, which follows from Lemma 4.3 .

Corollary 5.7 It is decidable whether a given finite monoid $M$ belongs to the pseudovariety $\left(\mathrm{B}_{1}(\mathrm{~m}) \mathrm{SI}\right) \cap \mathrm{F}$.

Proof: It is decidable whether $M$ belongs to the pseudovariety $\mathrm{B}_{1} m$ SI. Indeed, Straubing's description [26, 27] of this upper bound is obviously decidable; alternatively using the description by the Mal'cev product, the decidability result is contained in [16]. Hence the statement follows from Theorem 5.6. 


\section{The new versus the old upper bound}

By [3] there is a monoid $M \in \mathrm{B}_{1} m$ SI such that $M \notin \mathrm{F}$. This means $\left(\mathrm{B}_{1}(m) \mathrm{SI}\right) \not \subset \mathrm{F}$. In this section we exhibit a monoid $N \in \mathrm{F}$ such that $N \notin \mathrm{B}_{1}(m)$ Sl.

Let $A=\{a, b\}$ and $L$ be the language of all words that after every factor $a^{2}$ contain a factor $b^{2}$. This means that $L=\left(A^{*} a^{2}\left(A^{*} b^{2} A^{*}\right)^{c}\right)^{c}$, where the exponent $c$ stands for complementation in $A^{*}$. Note that the language $A^{*} b^{2} A^{*}=A^{*} b \emptyset^{*} b A^{*}$ is of level 2 . Hence $\left(A^{*} b^{2} A^{*}\right)^{c}$ is also of level 2 and consequently $L$ is a language of level 3 . We consider the syntactic monoid $N=M_{L}$ of this language and show that $N \notin \mathrm{B}_{1}\left(\mathrm{~m}\right.$ SI and that $N \in \mathrm{F}$. Consequently, $N \notin \mathrm{V}_{2}$, so that $L$ is not in level 2 .

In order to compute $N$, we first note that the minimal automaton of $L$ is the one described in Figure 1(a), where 1 is the initial state and 1 and 2 are the final states. It is then routine to compute the presentation

$$
N=\left\langle a, b: a b a=a, b a b=b, a^{3}=b a^{2}=a^{2}, a b^{2}=b^{3}=b^{2}\right\rangle
$$

and the eggbox picture of the monoid $N$, which is represented in Figure 1(b), where the *'s mark the idempotents.

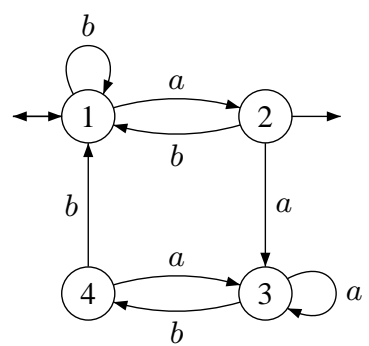

(a)

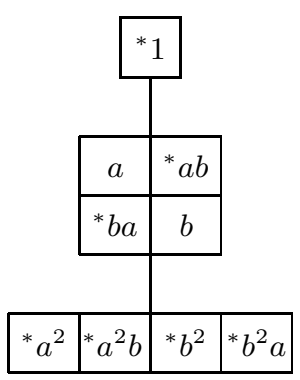

(b)

Fig. 1: Structural information about the language $L$

We show that $N \notin \mathrm{B}_{1}(\mathrm{~m}) \mathrm{SI}$. It is known (see e.g. (8.1) at page 732 in [13) that $\mathrm{B}_{1}(m) \mathrm{SI}$ is defined by all pseudoidentities

$$
\left(u^{\omega} w_{1} v^{\omega} w_{2} u^{\omega}\right)^{\omega} w_{1} v^{\omega} w_{4}\left(u^{\omega} w_{3} v^{\omega} w_{4} u^{\omega}\right)^{\omega}=\left(u^{\omega} w_{1} v^{\omega} w_{2} u^{\omega}\right)^{\omega}\left(u^{\omega} w_{3} v^{\omega} w_{4} u^{\omega}\right)^{\omega}
$$

where $u, v, w_{i} \in F_{A}(i=1, \ldots, 4)$ are such that $\alpha(u)=\alpha(v)=\alpha\left(w_{1}\right)=\alpha\left(w_{2}\right)=\alpha\left(w_{3}\right)=\alpha\left(w_{4}\right)$. We consider such a pseudoidentity where we put $u=v=w_{3}=w_{4}=(x y)^{\omega}, w_{1}=x y x^{2}$ and $w_{2}=y x y^{2}$ to obtain the pseudoidentity

$$
\left((x y)^{\omega} x y x^{2}(x y)^{\omega} y x y^{2}(x y)^{\omega}\right)^{\omega} x y x^{2}(x y)^{\omega}=\left((x y)^{\omega} x y x^{2}(x y)^{\omega} y x y^{2}(x y)^{\omega}\right)^{\omega}
$$

which is satisfied in $\mathrm{B}_{1} \mathrm{~m}(\mathrm{SI}$. To check that $N$ fails the pseudoidentity (6.2), we simply evaluate $x=a$ and $y=b$ in $N$, so that the left hand side of 6.2) evaluates to $a^{2} b$ while the right hand side gives $b^{2}$.

It remains to show that $N \in \mathrm{F}$. To prove it, in view of Theorem 5.5, it suffices to show that $s^{\omega}=$ $s^{\omega} t s^{\omega}$ for every pair $(s, t) \in T_{N}$. Recall that $T_{N}$ is the transitive closure of the submonoid $\left\langle S_{N}\right\rangle$ of the 
monoid $N \times N$, where $S_{N}$ is defined in $(4.2)$. We start by noting that the relation $\equiv^{N}$ is an equivalence relation with two classes, namely $\{1\}$ and $N \backslash\{1\}$. Taking into account that the only idempotents in the non-singleton $\equiv^{N}$-class that are not right zeros are $a b$ and $b a$, we conclude that the only non-diagonal pairs in $S_{N}$ are the following: $\left(b^{2}, a b\right),\left(a^{2} b, a b\right),\left(a^{2}, b a\right)$, and $\left(b^{2} a, b a\right)$. Hence all non-diagonal pairs $(s, t) \in T_{N}$ are such that $s$ is a right zero, and so $s^{\omega}=s^{\omega} t s^{\omega}$, thereby showing that $N \in \mathrm{F}$.

The constructed monoid $N$ and its properties established above yield the following statement.

Proposition 6.1 F $\nsubseteq \mathrm{B}_{1}$ m SI.

From the example from [3] and Proposition 6.1 we know that $\left(\mathrm{B}_{1} \mathrm{~m} \mathrm{SI}\right) \cap \mathrm{F}$ is a smaller pseudovariety of monoids than each of $B_{1} m$ SI and $F$. Hence $\left(B_{1} m S I\right) \cap F$ is currently the best known decidable upper bound for $\mathrm{V}_{2}$. We leave as an open question whether $\left(\mathrm{B}_{1}\right.$ m $\left.\mathrm{SI}\right) \cap \mathrm{F}=\mathrm{V}_{2}$.

\section{Acknowledgements}

The authors wish to thank the referees, whose comments and suggestions helped to improve readability of the paper.

The work of the first author was supported, in part, by Fundação para a Ciência e a Tecnologia (FCT) through the Centro de Matemática da Universidade do Porto and by the FCT project PTDC/MAT/65481 12006, which is partly funded by the European Community Fund FEDER. The work of the second author was supported, in part, by the Ministry of Education of the Czech Republic under the project MSM 0021622409 and by the Grant no. 201/09/1313 of the Grant Agency of the Czech Republic. The work leading to this paper has also been carried out within the framework of the ESF programme "Automata: from Mathematics to Applications (AutoMathA)", benefiting in particular from an exchange grant for the visit of the second author to the University of Porto.

\section{References}

[1] J. Almeida, Finite semigroups and universal algebra, World Scientific, Singapore, 1995, English translation.

[2] , Profinite semigroups and applications, Structural Theory of Automata, Semigroups, and Universal Algebra (New York) (Valery B. Kudryavtsev and Ivo G. Rosenberg, eds.), NATO Science Series II: Mathematics, Physics and Chemistry, vol. 207, Springer, 2005, Proceedings of the NATO Advanced Study Institute on Structural Theory of Automata, Semigroups and Universal Algebra, Montréal, Québec, Canada, 7-18 July 2003, pp. $1-45$.

[3] J. Almeida and O. Klíma, A counterexample to a conjecture concerning concatenation hierarchies, Inform. Process. Lett. 110 (2009), 4-7.

[4] Mu. Arfi, Opérations polynomiales et hiérarchies de concaténation, Theor. Comp. Sci. 91 (1991), 71-84.

[5] M. J. Branco and J.-E. Pin, Equations for the polynomial closure, ICALP 2009, Part II (Berlin) (S. Albers, A. Marchetti-Spaccamela, Y. Matias, S. Nikoletseas, and W. Thomas, eds.), Lecture Notes in Comput. Sci., vol. 5556, Springer, 2009, pp. 115-126.

[6] D. Cowan, Inverse monoids of dot-depth two, Int. J. Algebra Comput. 3 (1993), 411-424.

[7] _ A result on the dot-depth hierarchy for inverse monoids, Semigroups, Automata and Languages (Singapore) (J. Almeida, G. M. S. Gomes, and P. V. Silva, eds.), World Scientific, 1996, pp. 41-57. 
[8] S. Eilenberg, Automata, languages and machines, vol. B, Academic Press, New York, 1976.

[9] O. Klíma and L. Polák, Polynomial operators on classes of regular languages, CAI '09: Proceedings of the 3rd International Conference on Algebraic Informatics (Berlin, Heidelberg), Lecture Notes in Comput. Sci., no. 5725, Springer-Verlag, 2009, pp. 260-277.

[10] M. Kufleitner, The height of factorization forests, Proc. MFCS'08, Lecture Notes in Comput. Sci., no. 5162, 2008, pp. 443-454.

[11] V. A. Molchanov, Nonstandard characterization of pseudovarieties, Algebra Universalis 33 (1995), 533-547.

[12] On nonstandard axiomatization of elementarily non-axiomatizable classes of discrete algebraic systems, Sibirsk. Mat. Zh. 40 (1999), no. 2, 421-433, iv, translation in Siberian Math. J. 40 (1999), no. 2, 363-373.

[13] J.-E. Pin, Syntactic semigroups, Handbook of Formal Languages (G. Rozenberg and A. Salomaa, eds.), Springer, 1997.

[14] _ Algebraic tools for the concatenation product, Theor. Comp. Sci. 292 (2003), 317-342.

[15] J.-E. Pin and H. Straubing, Monoids of upper triangular matrices, Semigroups: structure and universal algebraic problems (Amsterdam) (G. Pollák, ed.), North-Holland, 1985, pp. 259-272.

[16] J.-E. Pin and P. Weil, Profinite semigroups, mal'cev products and identities, J. Algebra 182 (1996), 604-626.

[17] _ A Reiterman theorem for pseudovarieties of finite first-order structures, Algebra Universalis 35 (1996), $577-595$.

[18] _ Polynomial closure and unambiguous product, Theory Comput. Syst. 30 (1997), 383-422.

[19] _ A conjecture on the concatenation product, Theor. Inform. Appl. 35 (2001), 597-618 (2002), A tribute to Aldo de Luca.

[20] J. Reiterman, The Birkhoff theorem for finite algebras, Algebra Universalis 14 (1982), 1-10.

[21] J. Rhodes and B. Steinberg, The q-theory of finite semigroups, Springer Monographs in Mathematics, Springer, 2009.

[22] M. P. Schützenberger, On finite monoids having only trivial subgroups, Inform. and Control 8 (1965), 190-194.

[23] I. Simon, Factorization forests of finite height, Theor. Comp. Sci. 72 (1990), 65-94.

[24] The product of rational languages, 20th ICALP (Berlin), Lect. Notes Comput. Sci., vol. 700, Springer, 1993, pp. 430-444.

[25] H. Straubing, Finite semigroup varieties of the form $V * D$, J. Pure Appl. Algebra 36 (1985), 53-94.

[26] Semigroups and languages of dot-depth 2, Automata, languages and programming (Rennes, 1986), Lecture Notes in Comput. Sci., vol. 226, Springer, Berlin, 1986, pp. 416-423.

[27] _ Semigroups and languages of dot-depth two, Theor. Comp. Sci. 58 (1988), 361-378.

[28] H. Straubing and P. Weil, On a conjecture concerning dot-depth two languages, Theor. Comp. Sci. 104 (1992), 161-183.

[29] D. Thérien, Classification of finite monoids: the language approach, Theor. Comp. Sci. 14 (1981), 195-208. 